\title{
Solving Riemann-Hilbert problems with meromorphic functions
}

\section{Dan Kucerovsky}

Department of Mathematics, University of New Brunswick, Fredericton, NB, Canada email: dkucerov@unb.ca

\author{
Aydin Sarraf \\ Resson Aerospace Corporation, \\ Fredericton, NB, Canada \\ email: aydin.sarraf@resson.com
}

\begin{abstract}
In this paper, we introduce the use of a powerful tool from theoretical complex analysis, the Blaschke product, for the solution of Riemann-Hilbert problems. Classically, Riemann-Hilbert problems are considered for analytic functions. We give a factorization theorem for meromorphic functions over simply connected nonempty proper open subsets of the complex plane and use this theorem to solve RiemannHilbert problems where the given data consists of a meromorphic function.
\end{abstract}

\section{Introduction}

Approximation of holomorphic functions of a complex variable by a sequence of polynomials has a long history [23], some notable theorems in this regard are the Runge theorem [20], the Mergelyan theorem [19], and the Arakelyan theorem [2]. A different approach to approximation of a holomorphic function is to find and truncate an expansion or a factorization.

Since holomorphic functions are complex analytic, they admit Taylor expansion on an open disk. Furthermore, they admit Fourier expansions on the unit circle. Over the open unit disk, a holomorphic function can be written

2010 Mathematics Subject Classification: 35Q15, 30Dxx

Key words and phrases: Riemann-Hilbert problems, meromorphic functions, positive definite functions 
formally as a series of Blaschke products [9]. Moreover, entire functions can be factorized by Weierstrass factorization theorem [25]. In this paper, we give a factorization of meromorphic functions by Blaschke products over simply connected nonempty proper open subsets of the complex plane and use this theorem to solve Riemann-Hilbert problems with meromorphic functions.

One of the shortcomings of the classical solutions to Riemann-Hilbert problems is their dependence on the index of the coefficients and the Hölder continuity requirement in the application of Sokhotski-Plemelj formula. In [16], we proposed solutions to overcome these shortcomings. The current work can be considered as a sequel to [16], focusing on the complex variable case.

This paper is organized as follows. In Section 1, we define the classical Riemann-Hilbert problem, recall results on Blaschke products and state the Riemann Mapping Theorem. In Section 2, we use Blaschke products and the Riemann Mapping Theorem to give factorization theorems for meromorphic functions of bounded type over simply connected nonempty proper open subsets of the complex plane. In Section 3, we define a Riemann-Hilbert problem with meromorphic data and give a general solution by employing the results of Section 2. In Section 4, we give several results for positive definite functions on absolutely convex subsets of the complex plane. Our main results are Theorem (3), Theorem (4), Theorem (7) and their applications which are discussed in Section 3.

\subsection{Riemann-Hilbert problems with analytic functions}

The Riemann-Hilbert problem was first introduced by Bernhard Riemann in connection with the Riemann's Monodromy problem which later was generalized to the Riemann-Hilbert problem by Hilbert [1, A.1.3].

Definition 1 [10, 14.1.] Suppose that we are given a simple smooth closed contour $\mathrm{L}$ dividing the plane of the complex variable into an interior domain $\mathrm{D}^{+}$and an exterior domain $\mathrm{D}^{-}$, and two functions of on the contour, $\mathrm{G}(\mathrm{t})$ and $\mathrm{g}(\mathrm{t})$ which satisfy the Hölder condition, where $\mathrm{G}(\mathrm{t})$ does not vanish. It is required to find two functions: $\Phi^{+}(z)$, analytic in the domain $\mathrm{D}^{+}$; and $\Phi^{-}(z)$, analytic in the domain $\mathrm{D}^{-}$, including $z=\infty$, which satisfy on the contour $\mathrm{L}$ either the linear relation

$$
\Phi^{+}(z)=\mathrm{G}(\mathrm{t}) \Phi^{-}(z)
$$

or

$$
\Phi^{+}(z)=\mathrm{G}(\mathrm{t}) \Phi^{-}(z)+\mathrm{g}(\mathrm{t})
$$


The function $\mathrm{G}(\mathrm{t})$ will be called the coefficient of the Riemann problem, and the function $\mathrm{g}(\mathrm{t})$ its free (inhomogeneous) term.

The following theorem is of particular importance in the solution of analytic Riemann-Hilbert problems.

Theorem 1 [10, 13.2, Generalized Liouville's Theorem] Let the function $\mathbf{f}(z)$ be analytic in the entire complex plane, except at the points $\mathrm{a}_{0}=\infty, \mathrm{a}_{\mathrm{k}}(\mathrm{k}:=$ $1,2, \ldots, n)$, where it has poles, and suppose that the principal parts of the expansions of the function $\mathrm{f}(z)$ in the vicinities of the poles have the form:

at the point $\mathrm{a}_{0}$

$$
\mathrm{G}_{0}(z)=\mathrm{c}_{1}^{0} z+\mathrm{c}_{2}^{0} z^{2}+\ldots+\mathrm{c}_{\mathrm{n}_{0}}^{0} z^{\mathrm{n}_{0}}
$$

at the point $\mathrm{a}_{\mathrm{k}}$

$$
G_{0}\left(\frac{1}{z-a_{k}}\right)=\frac{c_{1}^{k}}{z-a_{k}}+\frac{c_{2}^{k}}{\left(z-a_{k}\right)^{2}}+\ldots+\frac{c_{m_{k}}^{k}}{\left(z-a_{k}\right)^{m_{k}}}
$$

Then the function $\mathrm{f}(\boldsymbol{z})$ is a rational function and is representable by the relation

$$
f(z)=C+G_{0}(z)+\sum_{k=1}^{n} G_{k}\left(\frac{1}{z-a_{k}}\right) .
$$

In particular, if the only singularity of the function $\mathrm{f}(z)$ is a pole of order $\mathrm{m}$ at infinity, then $\mathrm{f}(z)$ is a polynomial of degree $\mathrm{m}$ :

$$
f(z)=c_{0}+c_{1} z+\ldots+c_{m} z^{m} .
$$

\subsection{Blaschke products}

Definition 2 [11] A Blaschke product is a function of the form

$$
\mathrm{B}(z)=e^{i \alpha} z^{\mathrm{K}} \prod_{n \geq 1} \frac{\left|z_{\mathfrak{n}}\right|}{z_{\mathfrak{n}}} \frac{z_{n}-z}{1-\bar{z}_{\mathrm{n}} z}
$$

in which $\alpha \in \mathbb{R}, \mathrm{K} \in \mathbb{N}_{0}$, and $\left\{z_{1}, z_{2}, \ldots\right\}$ is a sequence (finite or infinite) in $\{0<|z|<1\}$ that satisfies the Blaschke condition

$$
\sum_{n \geq 1}\left(1-\left|z_{n}\right|\right)<\infty
$$


Finite Blaschke products can be considered as generalizations of polynomials in the unit disk because of their remarkable similar properties to polynomials $[18$, p. 249]. We only mention few of these similarities:

Proposition 1 The following hold:

(i) Let $\mathrm{f}$ be analytic in $\mathbb{C}$ and suppose that $\lim _{|z| \rightarrow \infty}|\mathrm{f}(z)|=\infty$ then $\mathrm{f}$ is a polynomial [18, Theorem 3].

(ii) Let $\mathrm{f}$ be analytic in $\mathbb{D}$ and suppose that $\lim _{|z| \rightarrow 1}|\mathrm{f}(z)|=1$ then $\mathrm{f}$ is a finite Blaschke product [18, Theorem 13].

(iii) Let $\mathrm{P}$ be a polynomial of degree $\mathrm{n}$ with zeros $z_{1}, \ldots, z_{\mathfrak{n}}$ in $\mathbb{C}$. The critical points of $\mathrm{P}$ lie in the convex hull of the set $\left\{z_{1}, \ldots, z_{n}\right\}[18$, Theorem 9].

(iv) Let $\mathrm{B}$ be a finite Blaschke product of degree $\mathrm{n}$ with zeros $z_{1}, \ldots, z_{\mathrm{n}}$ in $\mathbb{D}$. Then $\mathrm{B}(z)$ has exactly $\mathrm{n}-1$ critical points in $\mathbb{D}$ and these all lie in the hyperbolic convex hull ${ }^{1}$ of the set $\left\{z_{1}, \ldots, z_{n}\right\}[18$, Theorem 19].

\subsection{Riemann Mapping Theorem}

We recall the Riemann Mapping Theorem.

Theorem $2[3,14.2]$ For any simply connected domain $\mathrm{R}(\neq \mathbb{C})$ and $z_{0} \in \mathbb{R}$, there exists a unique conformal mapping $\phi$ of $\mathrm{R}$ onto $\mathrm{U}$ such that $\phi\left(z_{0}\right)=0$ and $\phi^{\prime}\left(z_{0}\right)>0$.

Example 1 The map $\mathrm{f}(z)=\frac{z-i}{z+i}$ is a conformal map of the unit disk to the upper half plane $\mathbb{H}$. In fact, all conformal maps from the upper half plane to the unit disk take the form $e^{i \theta} \frac{z-\beta}{z-\bar{\beta}}$ where $\theta \in \mathbb{R}$ and $\beta \in \mathbb{H}[22$, Chapter 8, Exercise 14].

For simple domains such as polygons, one can construct a Riemann map by using the Schwarz-Christoffel formula. The construction of a Riemann map for a general simply connected domain has been studied extensively and numerous algorithms are known $\mathrm{rm}[13,6,5,8,7]$.

\footnotetext{
${ }^{1}$ Recall that the Poincaré disk provides a model of the hyperbolic plane in the disk $|z|<1$; we refer to a line in the Poincaré model as a hyperbolic line and to the associated subregions as hyperbolic half-planes. The hyperbolic convex hull of a point set is the intersection of all hyperbolic half-planes containing the point set [24].
} 


\section{Factorization of meromorphic functions}

In this section, we give some theorems on factorization of meromorphic functions satisfying certain boundedness conditions in terms of (finite or infinite) Blaschke products.

Lemma 1 Let $\mathrm{f}: \mathrm{X} \subset \mathbb{C} \rightarrow \mathbb{C}$ be a holomorphic function where $\mathrm{X}$ is a simply connected bounded open set. If $\lim _{|z| \rightarrow|\mathrm{a}|}|\mathrm{f}(z)| \neq 0$ for all $\mathrm{a} \in \partial \mathrm{X}$, then $\mathrm{f}$ has finitely many zeros in $\mathrm{X}$.

Proof. Since $f$ is holomorphic on $X$, it is continuous on $X$. Assume that $f$ has infinitely many zeros. The zero set $Z=\left\{z_{k}\right\}$ of $f$ is bounded; therefore, it has an accumulation point by the Bolzano-Weierstrass Theorem. The accumulation point of zeros of $f$ does not belong to $\partial X$ because $\lim _{k \rightarrow \infty}\left|f\left(z_{k}\right)\right|=0$ but $\lim _{|z| \rightarrow|a|}|f(z)| \neq 0$. Therefore, the accumulation point must belong to $X$. By the Identity Theorem, $f \equiv 0$, on $X$ which is a contradiction.

Lemma 2 Let $\mathrm{f}: \mathrm{X} \subset \mathbb{C} \rightarrow \mathbb{C}$ be holomorphic on $\mathrm{X}$ where $\mathrm{X}$ is a simply connected open set. If $\mathrm{f}$ has no zeros in $\mathrm{X}$, then there exists a holomorphic function $\mathrm{h}$ on $\mathrm{X}$ such that $\mathrm{f}=\mathrm{e}^{\mathrm{h}}$. Furthermore, if $\mathrm{X}$ is bounded, $\mathrm{f}$ is continuous on $\overline{\mathrm{X}}$, and constant on $\partial \mathrm{X}$ then $\mathrm{f}$ is constant on $\mathrm{X}$.

Proof. The first part of the lemma is a standard result and its proof can be found in [17, XIII, Theorem 2.1]. For the second part, we note that if $f$ has no zeros in $X$, then $\frac{1}{f}$ is holomorphic on $X$. By the maximum modulus principle, the maximum of the harmonic function $\frac{1}{|f(z)|}$ is on the boundary of $X$. But also the maximum of the $|f|$ is on the boundary. If $|f|$ is constant on the boundary then $|\mathrm{f}(z)|=\mathrm{c}$ for all $z \in \mathrm{X}$.

Theorem 3 Let $\mathrm{f}: \mathrm{X} \subset \mathbb{C} \rightarrow \mathbb{C}$ be a meromorphic function where $\mathrm{X}$ is a simply connected bounded open Jordan domain. If $\lim _{|\mathrm{x}| \rightarrow|\mathrm{a}|}|\mathrm{f}(\mathrm{x})|$ where $\mathrm{a} \in \partial \mathrm{X}$ exists and it is not zero or infinity, then

$$
f(\phi(z))=e^{\mathrm{q}(z)} \prod_{i=1}^{n} \frac{z_{i}-z}{1-\overline{z_{i} z}} \prod_{j=1}^{m} \frac{\overline{p_{j}}-\frac{1}{z}}{1-\frac{p_{j}}{z}}
$$

where $\phi: \mathbb{D} \rightarrow \mathrm{X}$ is a Riemann map, $\mathrm{q}: \mathbb{D} \rightarrow \mathbb{C}$ is a holomorphic function, $\left\{z_{i}\right\}_{i=1}^{n}$ is the set of zeros and $\left\{p_{j}\right\}_{j=1}^{m}$ is the set of poles of $\mathrm{f} \circ \phi$. 
Proof. By the Riemann mapping theorem, there exists a conformal bijective map $\phi: \mathbb{D} \rightarrow X$. By Carathéodory's theorem, there exists a homeomorphism $\tilde{\phi}: \overline{\mathbb{D}} \rightarrow \bar{X}$ that extends $\phi$. Therefore, if $|z| \rightarrow|1|$ then $|\phi(z)| \rightarrow|\mathrm{a}|$ where $a \in \partial X$ and hence $\lim _{|z| \rightarrow 1}|f(\phi(z))| \neq 0, \infty$. Since $g=f \circ \phi: \mathbb{D} \rightarrow \mathbb{C}$ is meromorphic, it is the ratio of two holomorphic functions, i.e. $g=\frac{h}{k}$ where $h$ and $k$ are holomorphic. Since $\lim _{|z| \rightarrow|1|}|g(z)| \neq 0, \infty$, we conclude $\lim _{|z| \rightarrow|1|}|h(z)| \neq 0$ and $\lim _{|z| \rightarrow|1|}|k(z)| \neq 0$. By Lemma (1), $h$ and $k$ have finitely many zeros in $\mathbb{D}$, denoted by $\left\{z_{i}\right\}_{i=1}^{n}$ and $\left\{p_{j}\right\}_{j=1}^{m}$ respectively.

The function $h_{n}:=\frac{h}{B_{h}}$ where $B_{h}(z)=\prod_{i=1}^{n} \frac{z_{i}-z}{1-z_{i} z}$, is holomorphic in $\mathbb{D}$ and has no zeros in $\mathbb{D}$. By Lemma (2), there exists a holomorphic function $q_{h}$ such that $h_{h}=e^{q_{h}}$. Therefore, $h=e^{q_{h}} B_{h}$ and we can proceed similarly to prove $k=e^{q_{k}} B_{k}$. Hence, $g=e^{q_{h}-q_{k}} \frac{B_{h}}{B_{k}}$. Since $\overline{B_{k}}\left(\frac{1}{\bar{z}}\right)=\frac{1}{B_{k}(z)}$, we have $g(z)=e^{q(z)} B_{h}(z) \overline{B_{k}}\left(\frac{1}{\bar{z}}\right)$ where $q(z)=q_{h}(z)-q_{k}(z)$.

Definition 3 A function defined on a simply connected open subset $\mathrm{X}$ of the complex plane is said to be of bounded type if it is equal to the ratio of two analytic functions bounded in $\mathrm{X}$. The class of all such functions is called the Nevanlinna class for $\mathrm{X}$.

Lemma 3 [22, p. 156] If $\mathrm{f}$ is holomorphic in the unit disk, bounded and not identically zero, and $z_{1}, z_{2}, \ldots, z_{n}, \ldots$ are its zeros $\left(\left|z_{k}\right|<1\right)$, then

$$
\sum_{n}\left(1-\left|z_{n}\right|\right)<\infty .
$$

Lemma $4[14$, p. 64$]$ Let $\left\{\alpha_{n}\right\}$ be a sequence of non-zeros complex numbers in the open unit disc $\mathbb{D}$. A necessary and sufficient condition that the infinite product

$$
\mathrm{B}(z)=\prod_{n=1}^{\infty}\left[\frac{\bar{\alpha}_{n}}{\left|\alpha_{n}\right|} \frac{\left(\alpha_{n}-z\right)}{\left(1-\bar{\alpha}_{n} z\right)}\right]
$$

should converge uniformly on compact subsets of the unit disc is that $\sum_{n=1}^{\infty}\left(1-\left|\alpha_{n}\right|\right)<\infty$. When this condition is satisfied, the product defines an inner function whose zeros are exactly $\alpha_{1}, \alpha_{2}, \ldots$

We now obtain a factorization theorem that has useful applications to the Riemann-Hilbert problem. This is discussed further in Section 3. 
Theorem 4 Let $\mathrm{f}: \mathrm{X} \subset \mathbb{C} \rightarrow \mathbb{C}$ be a meromorphic function where $\mathrm{X}$ is a simply connected open set. If $\mathrm{f}$ is of bounded type then

$$
f(\phi(z))=z^{r-s} q(z) \prod_{i=1}^{\infty} \frac{\bar{z}_{i}}{\left|z_{i}\right|} \frac{z_{i}-z}{1-\overline{z_{i} z}} \prod_{j=1}^{\infty} \frac{\left|p_{j}\right|}{p_{j}} \frac{\overline{p_{j}}-\frac{1}{z}}{1-\frac{p_{j}}{z}}
$$

where $\phi: \mathbb{D} \rightarrow \mathrm{X}$ is a Riemann map, $\mathrm{r}, \mathrm{s} \in \mathbb{N}_{0}$, $\mathrm{q}$ is a bounded holomorphic function without zeros, $\left\{z_{i}\right\}$ is the set of zeros and $\left\{\mathrm{p}_{j}\right\}$ is the set of poles of $f \circ \phi$.

Proof. By Riemann mapping theorem, there exists a conformal bijective map $\phi: \mathbb{D} \rightarrow X$. Since $g=f \circ \phi: \mathbb{D} \rightarrow \mathbb{C}$ is meromorphic of bounded type, it's the ratio of two bounded holomorphic functions, i.e. $g=\frac{h}{k}$ where $h$ and $k$ are holomorphic and bounded. The functions $h$ and $k$ can be factorized as $h(z)=z^{\mathrm{r}} h_{1}(z)$ and $k(z)=z^{s} k_{1}(z)$ where $h_{1}(0) \neq 0$ and $k_{1}(0) \neq 0$. Let $\left\{z_{i}\right\}$ and $\left\{p_{j}\right\}$ be the zeros of $h_{1}$ and $k_{1}$. By Lemma (3), $\sum_{i}\left(1-\left|z_{i}\right|\right)<\infty$ and $\sum_{j}\left(1-\left|p_{j}\right|\right)<\infty$. By Lemma (4), the following products are convergent:

$$
\begin{aligned}
& \mathrm{B}_{\mathrm{h}_{1}}(z)=\prod_{i=1}^{\infty}\left[\frac{\bar{z}_{\mathrm{i}}}{\left|z_{\mathrm{i}}\right|} \frac{\left(z_{i}-z\right)}{\left(1-\bar{z}_{\mathrm{i}} z\right)}\right] \\
& \mathrm{B}_{\mathrm{k}_{1}}(z)=\prod_{j=1}^{\infty}\left[\frac{\bar{p}_{j}}{\left|\mathrm{p}_{j}\right|} \frac{\left(p_{j}-z\right)}{\left(1-\bar{p}_{j} z\right)}\right]
\end{aligned}
$$

Hence, we can write $h_{1}(z)=u(z) B_{h_{1}}(z)$ and $k_{1}(z)=v(z) B_{k_{1}}(z)$ where $u(z)=\frac{h_{1}(z)}{B_{h_{1}}(z)}$ and $v(z)=\frac{k_{1}(z)}{B_{k_{1}}(z)}$ are bounded holomorphic functions. Therefore,

$$
f(\phi(z))=z^{r-s} \frac{u(z)}{v(z)} \frac{B_{h_{1}}(z)}{B_{k_{1}}(z)}=z^{r-s} q(z) B_{h_{1}}(z) \bar{B}_{k_{1}}\left(\frac{1}{\bar{z}}\right)
$$

where $\mathrm{q}(z)=\frac{\mathrm{u}(z)}{v(z)}$ is a bounded holomorphic function.

\section{Applications in Riemann-Hilbert problems with meromorphic functions}

In engineering, a transfer function is a representation of the relation between the input and output of a linear time-invariant (LTI) system and it is a primary tool in classical control engineering. In this section, we employ Theorem (4) to find the transfer function of a differential system. 
Lemma 5 [4, Theorem 5.1] Suppose $f: \mathbb{R} \rightarrow \mathbb{C}$ is continuous, $\operatorname{supp}(f) \subset$ $[M, \infty)$, and has exponential order $\mathrm{a}$, i.e. $|\mathrm{f}(\mathrm{t})| \leq \mathrm{K} \mathrm{e}^{\mathrm{at}}$ for all $\mathrm{t} \in \mathbb{R}$. Then the Laplace transform $\mathfrak{L}(\mathbf{f})(z):=\int_{-\infty}^{\infty} e^{-z \mathfrak{t}} \mathbf{f}(\mathrm{t}) \mathrm{dt}$ is holomorphic in the half plane $\{z \mid \mathfrak{R}(z)>a\}$. The derivative is

$$
(\mathfrak{L}(f))^{\prime}(z)=-\int_{-\infty}^{\infty} e^{-z \mathfrak{t}} \mathrm{tf}(\mathrm{t}) \mathrm{dt}
$$

and the Laplace transform satisfies the estimate

$$
|\mathfrak{L}(f)(z)| \leq K \frac{e^{M(a-\Re(z))}}{(\mathfrak{R}(z)-a)}, \mathfrak{R}(z)>a
$$

Remark 1 If $\mathfrak{R}(z)>a+\epsilon>a$ and $M>0$ where $\epsilon>0$, then $|\mathfrak{L}(f)(z)| \leq$ $\frac{\mathrm{K}}{\epsilon e^{\mathrm{Me}}}$, i.e. the Laplace transform is bounded.

Lemma 6 [21, Theorem 2.12] Suppose that $\mathrm{f}(\mathrm{t}), \mathrm{f}^{\prime}(\mathrm{t}), \ldots, \mathrm{f}^{(\mathrm{n}-1)}(\mathrm{t})$ are continuous on $(0, \infty)$ and of exponential order, while $\mathrm{f}^{(\mathrm{n})}(\mathrm{t})$ is piecewise continuous on $[0, \infty)$. Then $\mathfrak{L}\left(f^{(n)}(t)\right)=s^{n} \mathfrak{L}(f(t))-s^{n-1} f\left(0^{+}\right)-s^{n-2} f^{\prime}\left(0^{+}\right)-\ldots-f^{(n-1)}\left(0^{+}\right)$.

Theorem 5 Suppose $f_{k}, g_{k}: \mathbb{R} \rightarrow \mathbb{C}$ are continuous, have left bounded support on the positive real line, and have positive exponential orders $\mathrm{a}_{\mathrm{k}}$ and $\mathrm{b}_{\mathrm{k}}$. Furthermore, assume that $\mathrm{u}, \mathrm{y}: \mathbb{R} \rightarrow \mathbb{C}$ are $\mathrm{n}$-times continuously differentiable, with $\mathrm{n}$ th derivative of exponential order. Then the transfer function of the following differential system with zero initial conditions, i.e. $\mathrm{u}^{(\mathrm{k})}(0)=0$, $y^{(k)}(0)=0$,

$$
\sum_{k=0}^{n} f_{k}(t) * \frac{d^{k} u(t)}{d t^{k}}=\sum_{k=0}^{n} g_{k}(t) * \frac{d^{k} y(t)}{d t^{k}}
$$

is a meromorphic function of bounded type of the form

$$
\mathrm{T}(\phi(z))=z^{\mathrm{r}-\mathrm{s}} \mathrm{q}(z) \prod_{i=1}^{\infty} \frac{\bar{z}_{\mathrm{i}}}{\left|z_{\mathrm{i}}\right|} \frac{z_{\mathrm{i}}-z}{1-\overline{z_{\mathrm{i}}} z} \prod_{j=1}^{\infty} \frac{\left|\mathrm{p}_{\mathrm{j}}\right|}{\mathrm{p}_{\mathrm{j}}} \frac{\overline{p_{j}}-\frac{1}{z}}{1-\frac{\mathrm{p}_{\mathrm{j}}}{z}}
$$

where $\phi: \mathbb{D} \rightarrow X$ is defined by $\phi(z):=\frac{1+(z-\alpha)}{1-(z-\alpha)}$ where $X=\{z \in \mathbb{C} \mid \mathfrak{R}(z)>\alpha\}$, $\alpha=\min \left\{a_{k}, b_{k}\right\}+\epsilon, \epsilon>0$ is sufficiently small, $r, s \in \mathbb{N}_{0}, q$ is a bounded holomorphic function without zeros, $\left\{z_{i}\right\}$ is the set of zeros and $\left\{p_{j}\right\}$ is the set of poles of $\mathrm{T} \circ \phi$. The transfer function $\mathrm{T}: \mathrm{X} \rightarrow \mathbb{C}$ appears as the coefficient of the Riemann-Hilbert problem $\Phi^{+}(z)=\mathrm{G}(z) \Phi^{-}(z)$ where

$$
\mathrm{G}(z)=\frac{\mathrm{T}(\phi(z))}{\mathrm{q}(z)}
$$




$$
\Phi^{+}(z)=z^{r} \prod_{i=1}^{\infty} \frac{\bar{z}_{i}}{\left|z_{i}\right|} \frac{z_{i}-z}{1-\overline{z_{i} z}}
$$

and

$$
\Phi^{-}(z)=z^{s} \prod_{j=1}^{\infty} \frac{\bar{p}_{j}}{\left|p_{j}\right|} \frac{p_{j}-z}{1-\bar{p}_{j} z}
$$

Proof. The transfer function is defined as the ratio of the Laplace transform of the output signal to the Laplace transform of the input signal, i.e. $\mathrm{T}(\mathrm{s}):=$ $\frac{\mathfrak{L}(y)(s)}{\mathfrak{L}(u)(s)}$. If we take Laplace transform of the differential system, apply Lemma (6), and the properties of Laplace transform with respect to convolution and addition, we derive the following equation

$$
\left(\sum_{k=0}^{n} s^{k} \mathfrak{L}\left(f_{k}\right)(s)\right) \mathfrak{L}(u)(s)=\left(\sum_{k=0}^{n} s^{k} \mathfrak{L}\left(g_{k}\right)(s)\right) \mathfrak{L}(y)(s)
$$

Therefore, the transfer function is of the following form

$$
T(s)=\frac{\mathfrak{L}(y)(s)}{\mathfrak{L}(u)(s)}=\frac{\sum_{k=0}^{n} s^{k} \mathfrak{L}\left(f_{k}\right)(s)}{\sum_{k=0}^{n} s^{k} \mathfrak{L}\left(g_{k}\right)(s)}
$$

On the domain $X=\{z \in \mathbb{C} \mid \mathfrak{R}(z)>\alpha\}$, where $\alpha=\min \left\{a_{k}, b_{k}\right\}+\epsilon$, and $\epsilon>0$ is sufficiently small, the transfer function

$$
T(s)=\frac{\mathfrak{L}(y)(s)}{\mathfrak{L}(u)(s)}=\frac{\sum_{k=0}^{n} s^{k-n} \mathfrak{L}\left(f_{k}\right)(s)}{\sum_{k=0}^{n} s^{k-n} \mathfrak{L}\left(g_{k}\right)(s)}
$$

is a meromorphic function of bounded type by Lemma (5). It suffices to apply Theorem (4) to the transfer function $\mathrm{T}$.

\section{Positive definite functions of a complex variable}

In this section, we give some results on positive definite functions over absolutely convex subsets of $\mathbb{C}$. It is interesting to see whether one can factorize 
a meromorphic positive definite function; in the sense of Definition (4), such that all factors are positive definite. Unfortunately, it is difficult to determine positive definiteness of Blaschke products; even the determination of hermitianness is difficult because it requires finding all the zeros of the equation $\mathrm{B}(-z)-\overline{\mathrm{B}(z)}=0$. Nevertheless, we give a theorem (Theorem (7)) that can simplify the determination of positive definiteness for holomorphic hermitian functions.

Definition $4 A$ set $\mathrm{X} \subseteq \mathbb{C}$ is called absolutely convex if for any points $\mathrm{x}_{1}, \mathrm{x}_{2}$ in $\mathrm{X}$ and any numbers $\lambda_{1}, \lambda_{2}$ in $\mathbb{C}$ satisfying $\left|\lambda_{1}\right|+\left|\lambda_{2}\right| \leq 1$, the sum $\lambda_{1} \chi_{1}+\lambda_{2} \chi_{2}$ belongs to $\mathrm{X}$.

If $X \subseteq \mathbb{C}$ is absolutely convex then $r X$ is absolutely convex for all $r \in \mathbb{C}$.

Definition 5 A function $\mathrm{f}: \mathrm{X} \rightarrow \mathbb{C}$ is positive definite, where $\mathrm{X} \subseteq \mathbb{C}$ is absolutely convex, if $\sum_{j, k=1}^{n} f\left(\frac{x_{j}-x_{k}}{2}\right) \xi_{j} \bar{\xi}_{k} \geq 0$ for every choice of $x_{1}, \ldots, x_{n}$ in $\mathrm{X}$ and $\xi_{1}, \ldots, \xi_{n}$ in $\mathbb{C}$.

If we set $\omega^{*}=\left[\bar{\xi}_{1}, \ldots, \bar{\xi}_{n}\right]$ and $A=\left[f\left(\frac{x_{j}-x_{k}}{2}\right)\right]_{j, k}$ then we can rewrite the above condition as $\omega^{*} A \omega \geq 0$, i.e. $A$ is positive-semidefinite. In the following proposition, we review some of the properties of positive definite functions.

Proposition 2 If $\mathrm{f}: \mathrm{X} \rightarrow \mathbb{C}$ is positive definite, where $\mathrm{X} \subseteq \mathbb{C}$ is absolutely convex, then the following hold:

(i) $f(0) \geq 0, f\left(-\frac{z}{2}\right)=\bar{f}\left(\frac{z}{2}\right)$, and $\left|f\left(\frac{z}{2}\right)\right|^{2} \leq f(0)^{2}$.

(ii) If $\mathrm{f}, \mathrm{g}: \mathrm{X} \rightarrow \mathbb{C}$ are positive definite then $\mathrm{fg}$ and $\mathrm{c}_{1} \mathrm{f}+\mathrm{c}_{2} \mathrm{~g}$ where $\mathrm{c}_{1}, \mathrm{c}_{2} \in \mathbb{N}$ are positive definite.

(iii) If $\mathrm{X}=\mathbb{R}, \mathrm{f}$ and $\mathrm{g}$ are integrable and positive definite then $\mathrm{f} * \mathrm{~g}$ is positive definite.

(iv) If $\mathrm{X}=\mathbb{R}$, and $\mathrm{f}$ is integrable then $\mathrm{x}^{2 \mathrm{k}+1} \mathrm{f}(\mathrm{x})$ with $\mathrm{k} \in \mathbb{N}$ is not positive definite.

(v) If $\mathrm{X}=\mathbb{R}$, and $\mathrm{f}$ is $\mathrm{C}^{\mathrm{n}}$-differentiable then $\frac{\mathrm{d}^{\mathrm{n}} \mathrm{f}(\mathrm{x})}{\mathrm{d} \mathrm{x}^{\mathrm{n}}}$ is positive definite only if $\mathrm{n}=4 \mathrm{k}$ where $\mathrm{k} \in \mathbb{N}$.

(vi) If $\mathrm{X}=\mathbb{R}$, and $\mathrm{f}$ is integrable then $\mathrm{e}^{\mathrm{iax}} \mathrm{f}(\mathrm{x})$ is positive definite. 
Proof. (i) If we set $n=1$ in Definition (5) then $f(0)\left|\xi_{1}\right|^{2} \geq 0$ which implies $f(0) \geq 0$. If we set $n=2$ and consider the set of points $\{z, 0\}$ then $\alpha=$ $f(0)^{2}\left(\left|\xi_{1}\right|^{2}+\left|\xi_{2}\right|^{2}\right)+f\left(-\frac{z}{2}\right) \xi_{1} \bar{\xi}_{2}+f\left(\frac{z}{2}\right) \xi_{2} \bar{\xi}_{1} \geq 0$. Since $\alpha=\bar{\alpha}$, we have $f\left(-\frac{z}{2}\right)=$ $\bar{f}\left(\frac{z}{2}\right)$. Since the following matrix is positive-semidefinite, its determinant is nonnegative, i.e. $\left|f\left(\frac{z}{2}\right)\right|^{2} \leq f(0)^{2}$.

$$
A=\left(\begin{array}{cc}
f(0) & f\left(\frac{z}{2}\right) \\
f\left(\frac{-z}{2}\right) & f(0)
\end{array}\right)
$$

(ii) Since positive linear combination and product of positive definite functions correspond to positive linear combination and Hadamard product of positive-semidefinite matrices, positive-definiteness is preserved under these operations.

(iii) By convolution theorem $\widehat{f * g}=\hat{f} * \hat{g}$ which is positive by Bochner's theorem.

(iv) The Fourier transform of $x^{2 k+1} f(x)$ is $\left(\frac{i}{2 \pi}\right)^{2 k+1} \frac{d^{2 k+1} \hat{f}(\xi)}{d \xi^{2 k+1}}$ which is purely imaginary by Bochner's theorem.

(v) The Fourier transform of $\frac{d^{n} f(x)}{d x^{n}}$ is $(2 \pi i \xi)^{n} \hat{f}(\xi)$ which is positive only if $n=4 k, k \in \mathbb{N}$.

(vi) The Fourier transform of $e^{i a x} f(x)$ is $\hat{f}\left(\xi-\frac{a}{2 \pi}\right)$.

Proposition 3 If the Möbius transform $\mathrm{f}(z)=\frac{\mathrm{a} z+\mathrm{b}}{\mathrm{c} z+\mathrm{d}}$ is positive definite and not identically zero on $\mathbb{C} \cup\{\infty\}$ then $\mathrm{a}=0$.

Proof. Assume $a \neq 0$ then we can set $z=\frac{2 b}{a}$ in the condition $f\left(-\frac{z}{2}\right)=\bar{f}\left(\frac{z}{2}\right)$ which implies $b=0$. Therefore, the condition $\left|f\left(\frac{z}{2}\right)\right|^{2} \leq f(0)^{2}$ implies that $f$ is identically zero which is a contradiction.

Lemma 7 The function $\mathrm{f}(z)=\mathrm{e}^{\mathrm{ia} \frac{z_{\mathrm{k}}-z}{1-\bar{z}_{\mathrm{k}} z}}$ where $z_{\mathrm{k}}, z \in \mathbb{D}$, and $\mathrm{a} \in \mathbb{R}$ is not positive definite.

Proof. For the set $\left\{\frac{i e^{-i a}}{2}, 0\right\}$ in $\mathbb{D}$, the determinant of the associated matrix $A$ is $\frac{\left|z_{k}\right|^{4}-1}{4+\bar{z}_{k}^{2} e^{-2 i a}}$. The numerator of $\operatorname{det}(A)$ is negative for $z_{k} \in \mathbb{D}$, but the denominator is not negative for any value of $z_{k} \in \mathbb{D}$, and $a \in \mathbb{R}$. Therefore, $\operatorname{det}(A)$ is not nonegative for all $z_{\mathrm{k}} \in \mathbb{D}$, and $\mathrm{a} \in \mathbb{R}$.

Theorem 6 A conformal map from $\mathbb{D}$ to $\mathbb{D}$ is not positive definite.

Proof. A conformal map from $\mathbb{D}$ to $\mathbb{D}$ is of the form $e^{i a_{k} \frac{z_{k}-z}{1-\bar{z}_{k} z}}$ which is not positive definite by Lemma (7). 
Lemma 8 [15, Exercise 1.1.8], [12, Lemma 24] For any nontrivial holomorphic function $\mathrm{f}: \mathrm{U} \rightarrow \mathbb{C}$ where $\mathrm{U} \subset \mathbb{C}^{\mathfrak{n}}$ is open and connected, $\mathrm{U}-\mathrm{Z}(\mathrm{f})$ is connected and dense in $\mathrm{U}$ where $\mathrm{Z}(\mathrm{f})$ denotes the zero set of $\mathrm{f}$.

Theorem 7 Let $\mathrm{X} \subseteq \mathbb{C}$ be an absolutely convex simply connected set and $\mathrm{f}: \mathrm{X} \subseteq \mathbb{C} \rightarrow \mathbb{C}$ be a holomorphic hermitian, i.e. $\mathrm{f}(-\boldsymbol{z})=\overline{\mathbf{f}(z)}$, function. Define the function $\mathrm{W}_{\mathrm{n}}(\mathrm{f}): \mathrm{X}^{\mathrm{n}} \rightarrow \mathrm{M}_{\mathrm{n}}(\mathbb{C})$ by $\mathrm{W}_{\mathrm{n}}(\mathrm{f})(\mathrm{x})=\left[\mathrm{f}\left(\frac{\mathrm{x}_{\mathrm{j}}-\mathrm{x}_{\mathrm{k}}}{2}\right)\right]_{\mathrm{j}, \mathrm{k}}$. If there exists a point $\mathrm{x} \in \mathrm{X}^{\mathrm{n}}$ at which $\mathrm{W}_{\mathrm{n}}(\mathrm{f})$ is positive definite for all $\mathrm{n} \in \mathbb{N}$ then $\mathrm{f}$ is positive definite on $\mathrm{X}$.

Proof. For simplicity, we denote $W_{n}(f)$ by $g$. Since $\operatorname{det}(g)$ is a polynomial of $f$ and $f$ is holomorphic, $\operatorname{det}(g)$ is holomorphic. By Lemma (8), $S=\operatorname{supp}(\operatorname{det}(g))$ is connected, open and dense in $X^{n}$. Since $f$ is hermitian, $\operatorname{Spec}(g)=\left\{\lambda_{x} \in \mathbb{C} \mid \lambda_{x}\right.$ is an eigenvalue of $\left.g(x), x \in X^{\mathfrak{n}}\right\}$ is in $\mathbb{R}$. By definition of $S, g$ is invertible on $S$. Therefore, $0 \notin \operatorname{Spec}\left(g_{\mid S}\right)$. We claim that either $\operatorname{Spec}\left(g_{\mid S}\right) \subseteq \mathbb{R}_{+}$or $\operatorname{Spec}\left(g_{\mid S}\right) \subseteq$ $\mathbb{R}_{-}$. Assume otherwise, then there exist $x, y \in S$ such that $\lambda_{x} \in \mathbb{R}_{+}$and $\lambda_{y} \in \mathbb{R}_{-}$. Since $S$ is path connected, there exists a path in $S$ that connects $x$ to $y$ in $S$. But, there is no path that connects $\lambda_{x}$ to $\lambda_{y}$ because $0 \notin \operatorname{Spec}\left(g_{\mid S}\right)$ which gives a contradiction. If there exists a point $x_{0} \in X^{n}$ for which $g$ is positive definite, then either $x_{0} \in S$ or $x_{0}$ is a limit point of $S$ because $S$ is dense in $X^{n}$. In either case, $\operatorname{Spec}\left(g_{\mid S}\right) \subseteq \mathbb{R}_{+}$and by density of $S$ we conclude $\operatorname{Spec}(\mathrm{g}) \subseteq \mathbb{R}_{+}$for all $\mathrm{n} \in \mathbb{N}$, i.e. $f$ is positive definite on $X$.

\section{References}

[1] Mark J. Ablowitz and Peter A. Clarkson, Solitons, nonlinear evolution equations and inverse scattering, volume 149. Cambridge Univ. Press, 1991.

[2] Norair Unanovich Arakelian, Uniform approximation on closed sets by entire functions, Izv. Ross. Akad. Nauk Ser. Mat., 28 (5) (1964), 11871206.

[3] Joseph Bak and Donald J. Newman, Complex analysis, undergraduate texts in mathematics, 1997.

[4] Richard Beals, Advanced mathematical analysis: periodic functions and distributions, complex analysis, Laplace transform and applications, volume 12. Springer Science \& Business Media, 2013. 
[5] Ilia Binder, Mark Braverman, and Michael Yampolsky, On the computational complexity of the Riemann mapping, Ark. Mat., 45 (2) (2007), 221-239.

[6] Ilia Binder, Cristobal Rojas, and Michael Yampolsky, Computable caratheodory theory, Adv. Math., 265 (2014), 280-312.

[7] Christopher J. Bishop, A fast approximation to the Riemann map, preprint, 420, 2003.

[8] Christopher J. Bishop, Conformal mapping in linear time, Discrete Comput. Geom., 44 (2) (2010), 330-428

[9] Ronald R. Coifman and Stefan Steinerberger, Nonlinear phase unwinding of functions, J. Fourier Anal. Appl., 23 (4) (2017), 778-809.

[10] Fedor Dmitrievich Gakhov, Boundary value problems, volume 85, Pergamon Press, 1966.

[11] Stephan Ramon Garcia, Javad Mashreghi, and William T Ross, Finite Blaschke products: a survey, arXiv preprint arXiv:1512.05444, 2015.

[12] Paul M. Gauthier, Lectures on several complex variables, Springer, 2014.

[13] Peter Hertling, An effective Riemann mapping theorem, Theoret. Comput. Sci., 219 (1-2) (1999), 225-265.

[14] Kenneth Hoffman, Banach spaces of analytic functions, Courier Corporation, 2007.

[15] Daniel Huybrechts, Complex geometry: an introduction, Springer Science \& Business Media, 2006.

[16] Dan Kučerovskỳ, Amir TP Najafabadi, and Aydin Sarraf, On the riemann-hilbert factorization problem for positive definite functions, Positivity, 20 (3) (2016), 743-754.

[17] Serge Lang, Complex analysis, volume 103, Springer Science \& Business Media, 2013.

[18] Javad Mashreghi and Emmanuel Fricain, Blaschke products and their applications, Springer, 2013. 
[19] S. N. Mergelian, On the representation of functions by series of polynomials on closed sets, Number 85. Amer. Math. Soc., 1953.

[20] Carle Runge, Zur Theorie der eindeutigen analytischen Functionen, Acta Math., 6 (1) (1885), 229-244.

[21] Joel L. Schiff, The Laplace transform: theory and applications, Springer Science \& Business Media, 2013.

[22] Elias M. Stein and Rami Shakarchi, Complex analysis, Princeton lectures in analysis, ii, 2003.

[23] Anatoliy Georgievich Vitushkin, Uniform approximations by holomorphic functions, J. Funct. Anal., 20 (2) (1975), 149-157.

[24] J. L. Walsh, Note on the location of zeros of extremal polynomials in the non-euclidean plane, Acad. Serbe Sci. Publ. Inst. Math, 4 (1952), $157-160$.

[25] Carl Weierstrass, Zur Theorie der eindeutigen analytischen Functionen, na, 1877.

Received: April 5, 2018 